\title{
A NOTE ON CONTIGUITY AND HELLINGER DISTANCE*
}

\author{
by \\ J. OOSTERHOFF (1), AND W. R. VAN ZWET (2)
}

\section{Introduction}

For $n=1,2, \ldots$ let $\left(\mathscr{X}_{n 1}, \mathscr{A}_{n 1}\right), \ldots,\left(\mathscr{X}_{n n}, \mathscr{A}_{n n}\right)$ be arbitrary measurable spaces. Let $P_{n i}$ and $Q_{n i}$ be probability measures defined on $\left(\mathscr{X}_{n i}, \mathscr{A}_{n i}\right), i=1, \ldots, n$; $n=1,2, \ldots$, and let $P_{n}^{(n)}=\prod_{i=1}^{n} P_{n i}$ and $Q_{n}^{(n)}=\prod_{i=1}^{n} Q_{n i}(n=1,2, \ldots)$ denote the product probability measures. For each $i$ and $n$ let $X_{n i}$ be the identity map from $\mathscr{X}_{n i}$ onto $\mathscr{X}_{n i}$. Then $P_{n i}$ and $Q_{n i}$ represent the two possible distributions of the random element $X_{n i}$ as well as the probability measures of the underlying probability space. Obviously $X_{n 1}, \ldots, X_{n n}$ are independent under both $P_{n}^{(n)}$ and $Q_{n}^{(n)}(n=1,2, \ldots)$.

The sequence $\left\{Q_{n}^{(n)}\right\}$ is said to be contiguous with respect to the sequence $\left\{P_{n}^{(n)}\right\}$ if $\lim P_{n}^{(n)}\left(A_{n}\right)=0$ implies $\lim Q_{n}^{(n)}\left(A_{n}\right)=0$ for any sequence of measurable sets $A_{n}$. $\lim _{n \rightarrow \infty} P_{n}$ $n \rightarrow \infty$

This one-sided contiguity notion is denoted by $\left\{Q_{n}^{(n)}\right\} \triangleleft\left\{P_{n}^{(n)}\right\}$ (the notation is due to H. Witting \& G. Nölle [7]). The sequences $\left\{P_{n}^{(n)}\right\}$ and $\left\{Q_{n}^{(n)}\right\}$ are said to be contiguous with respect to each other if both $\left\{Q_{n}^{(n)}\right\} \triangleleft\left\{P_{n}^{(n)}\right\}$ and $\left\{P_{n}^{(n)}\right\} \triangleleft\left\{Q_{n}^{(n)}\right\}$. This two-sided contiguity concept we denote by $\left\{P_{n}^{(n)}\right\} \triangleleft \triangleright\left\{Q_{n}^{(n)}\right\}$.

The main purpose of this note is to characterize contiguity of product probability measures in terms of their marginals. To this end we introduce the Hellinger distance $H(P, Q)$ between two probability measures $P$ and $Q$ on the same $\sigma$-field, defined by

$$
H(P, Q)=\left\{\int\left(p^{1 / 2}-q^{1 / 2}\right)^{2} \mathrm{~d} \mu\right\}^{1 / 2}=\left\{2-2 \int p^{1 / 2} q^{1 / 2} \mathrm{~d} \mu\right\}^{1 / 2}
$$

where $p=\mathrm{d} P / \mathrm{d} \mu, q=\mathrm{d} Q / \mathrm{d} \mu$ and $\mu$ is any $\sigma$-finite measure dominating $P+Q$. This metric is independent of the choice of $\mu$ and satisfies $0 \leqq H(P, Q) \leqq 2^{1 / 2}$.

* Report SW 36/75 Mathematisch Centrum, Amsterdam

AMS (MOS) subject classification scheme (1970): 62E20

KEY WORDS \& PHRASES: asymptotic normality, contiguity, Hellinger distance, log likelihood ratio. 
Defining the total variation distance of $P$ and $Q$ by

$$
\|P-Q\|=\sup |P(A)-Q(A)|
$$

where the supremum is taken over all measurable sets $A$, we have the following inequalities (Le Cam [4])

$$
\frac{1}{2} H^{2}(P, Q) \leqq\|P-Q\| \leqq H(P, Q)
$$

The Hellinger distances of the product measures and of their marginals are connected by the relationship

$$
H^{2}\left(P_{n}^{(n)}, Q_{n}^{(n)}\right)=2-2 \prod_{i=1}^{n}\left\{1-\frac{1}{2} H^{2}\left(P_{n i}, Q_{n i}\right)\right\}
$$

For further reference we first mention two easy results, viz.

$$
\sum_{i=1}^{n} H^{2}\left(P_{n i}, Q_{n i}\right)=o(1) \text { for } n \rightarrow \infty \Rightarrow\left\{P_{n}^{(n)}\right\} \triangleleft \triangleright\left\{Q_{n}^{(n)}\right\},
$$

and

$$
\left\{Q_{n}^{(n)}\right\} \triangleleft\left\{P_{n}^{(n)}\right\} \Rightarrow \sum_{i=1}^{n} H^{2}\left(P_{n i}, Q_{n i}\right)=O(1) \text { for } n \rightarrow \infty .
$$

The proof of (1.5) is an immediate consequence of the string of implications

$$
\begin{gathered}
\sum_{i=1}^{n} H^{2}\left(P_{n i}, Q_{n i}\right)=o(1) \Rightarrow \sum_{i=1}^{n} \log \left\{1-\frac{1}{2} H^{2}\left(P_{n i}, Q_{n i}\right)\right\}=o(1) \\
\Rightarrow H^{2}\left(P_{n}^{(n)}, Q_{n}^{(n)}\right)=o(1) \Rightarrow\left\|P_{n}^{(n)}-Q_{n}^{(n)}\right\|=o(1) \Rightarrow\left\{P_{n}^{(n)}\right\} \triangleleft \triangleright\left\{Q_{n}^{(n)}\right\} .
\end{gathered}
$$

To prove (1.6) suppose that $\limsup _{n \rightarrow \infty} H\left(P_{n}^{(n)}, Q_{n}^{(n)}\right)=2^{1 / 2}$. Then by (1.3) $\limsup _{n \rightarrow \infty} \| P_{n}^{(n)}-$ $-Q_{n}^{(n)} \|=1$ in contradiction to $\left\{Q_{n}^{(n)}\right\} \triangleleft\left\{P_{n}^{(n)}\right\}$. Thus $\limsup _{n \rightarrow \infty} H^{2}\left(P_{n}^{(n)}, Q_{n}^{(n)}\right)<$ $<2$, therefore $\liminf _{n \rightarrow \infty} \prod_{i=1}^{n}\left\{1-\frac{1}{2} H^{2}\left(P_{n i}, Q_{n i}\right)\right\}>0$ and hence $\limsup _{n \rightarrow \infty} \sum_{i=1}^{n} H^{2}\left(P_{n i}, Q_{n i}\right)<$ $<\infty$ and the proof is complete.

It can be shown by counterexamples that in (1.5) the condition cannot be weakened to $\sum_{i=1}^{n} H^{2}\left(P_{n i}, Q_{n i}\right)=O(1)$, and that in (1.6) the conclusion cannot be strengthened to $\sum_{i=1}^{n} H^{2}\left(P_{n i}, Q_{n i}\right)=o(1)$, for $n \rightarrow \infty$. Hence there remains a gap between the sufficient condition and the necessary condition for contiguity in (1.5) and (1.6) respectively. In section 2 we obtain conditions which are both sufficient and necessary for contiguity of the product measures by adding another condition to $\sum_{i=1}^{n} H^{2}\left(P_{n \imath}, Q_{n i}\right)=O(1)$. 
In many applications asymptotic normality of the log likelihood ratio statistic $\Lambda_{n}$ (see (3.1)) plays an important part. Since

$$
\mathscr{L}\left(\Lambda_{n} \mid P_{n}^{(n)}\right) \rightarrow_{w} \mathscr{N}\left(-\frac{1}{2} \sigma^{2} ; \sigma^{2}\right) \text { implies } \quad\left\{P_{n}^{(n)}\right\} \triangleleft \triangleright\left\{Q_{n}^{(n)}\right\}
$$

(cf. Hájek \& Šidák [1], Le Cam [2], [3], [4], Roussas [6]), we have to impose stronger conditions on the marginals $P_{n i}$ and $Q_{n i}$ to ensure the asymptotic normality of $\Lambda_{n}$. Some sufficient (and almost necessary) conditions for the asymptotic normality of $\Lambda_{n}$, which are clearly stronger than those in section 2 , are given in section 3 . These conditions are closely related to some earlier results of Le Cam [3], [4].

\section{Contiguity of product measures}

We begin by noting the following useful implication:

$$
\left\{Q_{n}^{(n)}\right\} \triangleleft\left\{P_{n}^{(n)}\right\} \Rightarrow\left[\lim _{n \rightarrow \infty} \sum_{i=1}^{n} P_{n i}\left(A_{n i}\right)=0 \Rightarrow \lim _{n \rightarrow \infty} \sum_{i=1}^{n} Q_{n i}\left(A_{n i}\right)=0\right]
$$

for any collection of measurable sets $A_{n i}$. For suppose $\lim _{n \rightarrow \infty} \sum_{i=1}^{n} P_{n i}\left(A_{n i}\right)=0$. Then $\lim _{n \rightarrow \infty} P_{n}^{(n)}\left(\bigcup_{i=1}^{n} A_{n i}\right)=0$, hence by contiguity $\lim _{n \rightarrow \infty} Q_{n}^{(n)}\left(\bigcup_{i=1}^{n} A_{n i}\right)=1-\lim _{n \rightarrow \infty} \prod_{i=1}^{n}\left(1-Q_{n i}\left(A_{n i}\right)\right)$ $\stackrel{n \rightarrow \infty}{=} 0$ and therefore $\lim _{n \rightarrow \infty} \sum_{i=1}^{n} Q_{n i}\left(A_{n i}\right)=0$.

Now let $\mu_{n i}$ be a $\sigma$-finite measure on $\left(\mathscr{X}_{n i}, \mathscr{A}_{n i}\right)$ dominating $P_{n i}+Q_{n i}$ and write $p_{n i}=\mathrm{d} P_{n i} / \mathrm{d} \mu_{n i}$ and $q_{n i}=\mathrm{d} Q_{n i} / \mathrm{d} \mu_{n i}(i=1, \ldots, n ; n=1,2, \ldots)$. The main result of this section is

Theorem 1. $\left\{Q_{n}^{(n)}\right\} \triangleleft\left\{P_{n}^{(n)}\right\}$ iff

$$
\limsup _{n \rightarrow \infty} \sum_{i=1}^{n} H^{2}\left(P_{n i}, Q_{n i}\right)<\infty
$$

and

$$
\lim _{n \rightarrow \infty} \sum_{i=1}^{n} Q_{n i}\left(q_{n i}\left(X_{n i}\right) / p_{n i}\left(X_{n i}\right) \geqq c_{n}\right)=0 \quad \text { whenever } \quad c_{n} \rightarrow \infty .
$$

Proof. First assume that (2.2) and (2.3) are satisfied. Write

$$
L_{n i}=q_{n i}\left(X_{n i}\right) / p_{n i}\left(X_{n i}\right), \quad i=1, \ldots, n ; \quad n=1,2, \ldots,
$$

and consider $\prod_{i=1}^{n} L_{n i}$. It is easily shown (cf. Le Cam [4], Roussas [6]) that $\left\{Q_{n}^{(n)}\right\} \triangleleft$ $\triangleleft\left\{P_{n}^{(n)}\right\}$ is equivalent to tightness of the sequence of distributions $\left\{\mathscr{L}\left(\prod_{i=1}^{n} L_{n i} \mid Q_{n}^{(n)}\right)\right.$; 
$n=1,2, \ldots\}$. The tightness of this set of distributions can also be expressed in the more convenient form

$$
\lim _{n \rightarrow \infty} Q_{n}^{(n)}\left(\prod_{i=1}^{n} L_{n i} \geqq k_{n}\right)=0 \quad \text { whenever } \quad k_{n} \rightarrow \infty
$$

Hence we have to prove (2.4). Let $0<k_{n} \rightarrow \infty$. Let $0<c_{n} \rightarrow \infty$ be real numbers to be chosen in the sequel. If $1_{A}$ denotes the indicator function of the set $A$, we have by (2.3) and Markov's inequality for $n \rightarrow \infty$

$$
\begin{gathered}
Q_{n}^{(n)}\left(\prod_{i=1}^{n} L_{n i} \geqq k_{n}\right) \\
\leqq Q_{n}^{(n)}\left(\prod_{i=1}^{n} L_{n i} \geqq k_{n} \wedge L_{n i}<c_{n} \quad \text { for } \quad i=1, \ldots, n\right)+Q_{n}^{(n)}\left(\bigcup_{i=1}^{n}\left\{L_{n i} \geqq c_{n}\right\}\right) \\
\leqq Q_{n}^{(n)}\left(\prod_{i=1}^{n} L_{n i}^{1 / 2} 1_{\left(0, c_{n}\right)}\left(L_{n i}\right) \geqq k_{n}^{1 / 2}\right)+\sum_{i=1}^{n} Q_{n i}\left(L_{n i} \geqq c_{n}\right) \\
\leqq k_{n}^{-1 / 2} \prod_{i=1}^{n} \int_{q_{n i}<c_{n} p_{n i}} q_{n i}^{3 / 2} p_{n i}^{-1 / 2} \mathrm{~d} \mu_{n i}+o(1) .
\end{gathered}
$$

Since for all $c_{n} \geqq 1$

$$
\begin{gathered}
\int_{q_{n i}<c_{n} p_{n i}} q_{n i}^{3 / 2} p_{n i}^{-1 / 2} \mathrm{~d} \mu_{n i} \\
\leqq \int_{q_{n i}<c_{n} p_{n i}} q_{n i} \mathrm{~d} \mu_{n i}+\int_{q_{n i}<c_{n} p_{n i}} q_{n i} p_{n i}^{-1 / 2}\left(q_{n i}^{1 / 2}-p_{n i}^{1 / 2}\right) \mathrm{d} \mu_{n i} \\
\leqq 1+\int_{q_{n i}<c_{n} p_{n i}} q_{n i}^{1 / 2} p_{n i}^{-1 / 2}\left(q_{n i}^{1 / 2}-p_{n i}^{1 / 2}\right)^{2} \mathrm{~d} \mu_{n i}+\int_{q_{n i}<c_{n} p_{n i}} q_{n i}^{1 / 2}\left(q_{n i}^{1 / 2}-p_{n i}^{1 / 2}\right) \mathrm{d} \mu_{n i} \\
\leqq 1+c_{n}^{1 / 2} \int\left(q_{n i}^{1 / 2}-p_{n i}^{1 / 2}\right)^{2} \mathrm{~d} \mu_{n i}+1-\int q_{n i}^{1 / 2} p_{n i}^{1 / 2} \mathrm{~d} \mu_{n i} \\
-\int_{q_{n i} \geqq c_{n} p_{n i}} q_{n i}^{1 / 2}\left(q_{n i}^{1 / 2}-p_{n i}^{1 / 2}\right) \mathrm{d} \mu_{n i} \leqq 1+\left(c_{n}^{1 / 2}+\frac{1}{2}\right) H^{2}\left(P_{n i}, Q_{n i}\right),
\end{gathered}
$$

it follows that

$$
\begin{gathered}
\limsup _{n \rightarrow \infty} Q_{n}^{(n)}\left(\prod_{i=1}^{n} L_{n i} \geqq k_{n}\right) \\
\leqq \limsup _{n \rightarrow \infty} k_{n}^{-1 / 2} \prod_{i=1}^{n}\left\{1+\left(c_{n}^{1 / 2}+\frac{1}{2}\right) H^{2}\left(P_{n i}, Q_{n i}\right)\right\} \\
\leqq \limsup _{n \rightarrow \infty} k_{n}^{-1 / 2} \exp \left\{\left(c_{n}^{1 / 2}+\frac{1}{2}\right) \sum_{i=1}^{n} H^{2}\left(P_{n i}, Q_{n i}\right)\right\}
\end{gathered}
$$


Choosing $c_{n}$ in such a way that $c_{n}=o\left(\left(\log k_{n}\right)^{2}\right)$ for $n \rightarrow \infty$, (2.2) implies $Q_{n}^{(n)}\left(\prod_{i=1}^{n} L_{n i} \geqq k_{n}\right)=o(1)$ for $n \rightarrow \infty$ and (2.4) is established.

Conversely, suppose that $\left\{Q_{n}^{(n)}\right\} \triangleleft\left\{P_{n}^{(n)}\right\}$. Since (1.6) implies that (2.2) is satisfied, it remains to prove (2.3). Let $0<c_{n} \rightarrow \infty$ and consider the inequality, valid for $c_{n} \geqq 4$,

$$
\begin{gathered}
\sum_{i=1}^{n} \int_{q_{n i} \geqq c_{n} p_{n i}} p_{n i} \mathrm{~d} \mu_{n i} \leqq c_{n}^{-1 / 2} \sum_{i=1}^{n} \int_{q_{n i} \geqq c_{n} p_{n i}} p_{n i}^{1 / 2} q_{n i}^{1 / 2} \mathrm{~d} \mu_{n i} \\
=c_{n}^{-1 / 2}\left\{\sum_{i=1}^{n} \int_{q_{n i} \geqq c_{n p_{n i}}} p_{n i}^{1 / 2}\left(q_{n i}^{1 / 2}-p_{n i}^{1 / 2}\right) \mathrm{d} \mu_{n i}+\sum_{i=1}^{n} \int_{q_{n i} \geqq c_{n} p_{n i}} p_{n i} \mathrm{~d} \mu_{n i}\right\} \\
\leqq c_{n}^{-1 / 2}\left\{\sum_{i=1}^{n} \int_{q_{n i} \geqq c_{n} p_{n i}}\left(q_{n i}^{1 / 2}-p_{n i}^{1 / 2}\right)^{2} \mathrm{~d} \mu_{n i}+\sum_{i=1}^{n} \int_{q_{n i} \geqq c_{n} p_{n i}} p_{n i} \mathrm{~d} \mu_{n i}\right\} \\
\leqq c_{n}^{-1 / 2}\left\{\sum_{i=1}^{n} H^{2}\left(P_{n i}, Q_{n i}\right)+\sum_{i=1}^{n} \int_{q_{n i} \geqq c_{n} p_{n i}} p_{n i} \mathrm{~d} \mu_{n i}\right\} .
\end{gathered}
$$

Since by $(2.2) c_{n}^{-1 / 2} \sum_{i=1}^{n} H^{2}\left(P_{n i}, Q_{n i}\right) \rightarrow 0$ for $n \rightarrow \infty$, it follows that $\lim _{n \rightarrow \infty} \sum_{i=1}^{n} P_{n}\left(L_{n i} \geqq\right.$ $\left.\geqq c_{n}\right)=0$. Hence $(2.1)$ implies that $\lim _{n \rightarrow \infty} \sum_{i=1}^{n} Q_{n i}\left(L_{n i} \geqq c_{n}\right)=0$ and the proof of the
theorem is complete. $\square$

Corollary 1. $\left\{P_{n}^{(n)}\right\} \triangleleft \triangleright\left\{Q_{n}^{(n)}\right\}$ iff (2.2) and (2.3) are satisfied and

$$
\lim _{n \rightarrow \infty} \sum_{i=1}^{n} P_{n i}\left(p_{n i}\left(X_{n i}\right) / q_{n i}\left(X_{n i}\right) \geqq c_{n}\right)=0 \quad \text { whenever } \quad c_{n} \rightarrow \infty \text {. }
$$

In connection with contiguity Hellinger distance seems to be a more appropriate metric than total variation distance. Note that from (1.3) and (1.6) we immediately obtain the implication

$$
\left\{Q_{n}^{(n)}\right\} \triangleleft\left\{P_{n}^{(n)}\right\} \Rightarrow \sum_{i=1}^{n}\left\|P_{n i}-Q_{n i}\right\|^{2}=O(1) \text { for } n \rightarrow \infty,
$$

where again the order term cannot be strenghtened to $o(1)$. However, $\sum_{i=1}^{n}\left\|P_{n i}-Q_{n i}\right\|^{2}=O(1)$ is too weak a condition to replace (2.2) in Theorem 1. On the other hand we cannot strengthen this condition to $\sum_{i=1}^{n}\left\|P_{n i}-Q_{n i}\right\| r=O(1)$ for some $r<2$, since $\left\{Q_{n}^{(n)}\right\} \triangleleft\left\{P_{n}^{(n)}\right\}$ does not necessarily imply $\sum_{i=1}^{n}\left\|P_{n i}-Q_{n i}\right\|^{r}=O(1)$ for any positive $r<2$. The following example serves to illustrate these points. 
Example. Let $\mu_{n i}$ denote Lebesgue measure on $(0,1)$, let $p_{n i}=1_{(0,1)}$ and let $q_{n i}=$ $=\left(1+n^{-1 / 2}\right) 1_{\left(0,1-n^{-1 / 2}\right)}+n^{-1 / 2} 1_{\left[1-n^{-1 / 2}, 1\right)}, i=1, \ldots, n ; n=1,2, \ldots$ Then $\sum_{i=1}^{n}\left\|P_{n t}-Q_{n i}\right\|^{2}=\left(1-n^{-1 / 2}\right)^{2} \leqq 1$ and $(2.3)$ is trivially satisfied since $q_{n i} / p_{n i}$ is uniformly bounded. But $\left\{Q_{n}^{(n)}\right\} \triangleleft\left\{P_{n}^{(n)}\right\}$ does not hold because $\sum_{i=1}^{n} H^{2}\left(P_{n t}, Q_{n i}\right)=$ $=2 n\left\{1-\int q_{n i}^{1 / 2} \mathrm{~d} \mu_{n i}\right\}=2 n\left\{1-\left(1+n^{-1 / 2}\right)^{1 / 2}\left(1-n^{-1 / 2}\right)-n^{-3 / 4}\right\}=n^{1 / 2}(1+$ $+o(1))$ for $n \rightarrow \infty$.

Taking $q_{n i}=\left(1+n^{-1 / 2}\right) 1_{(0,1 / 2)}+\left(1-n^{-1 / 2}\right) 1_{[1 / 2,1)}$ for all $i$ and $n$, we have $\left\{Q_{n}^{(n)}\right\} \triangleleft\left\{P_{n}^{(n)}\right\}$ since $(2.3)$ is satisfied and $\sum_{i=1}^{n} H^{2}\left(P_{n i}, Q_{n i}\right)=2 n\left\{1-\frac{1}{2}\left(1+n^{-1 / 2}\right)^{1 / 2}-\right.$ $\left.-\frac{1}{2}\left(1-n^{-1 / 2}\right)^{1 / 2}\right\}=\frac{1}{4}+o(1)$ for $n \rightarrow \infty$. However, in this case $\sum_{i=1}^{n}\left\|P_{n i}-Q_{n i}\right\| r=$ $=n\left(\frac{1}{2} n^{-1 / 2}\right)^{r} \rightarrow \infty$ for $n \rightarrow \infty$ if $r<2$.

\section{Asymptotic normality of $\Lambda_{n}$}

Define

$$
\Lambda_{n}=\sum_{i=1}^{n} \log \left\{q_{n i}\left(X_{n i}\right) / p_{n i}\left(X_{n i}\right)\right\}, \quad n=1,2, \ldots
$$

Note that, with probability one, $\Lambda_{n}$ is well-defined under $P_{n}^{(n)}$, although $\Lambda_{n}$ may assume the value $-\infty$ with positive probability under $P_{n}^{(n)}$.

In our search for necessary and sufficient conditions for the weak convergence $\mathscr{L}\left(\Lambda_{n} \mid P_{n}^{(n)}\right) \rightarrow_{w} \mathscr{N}\left(-\frac{1}{2} \sigma^{2} ; \sigma^{2}\right)$ in terms of the marginal distributions of the $X_{n i}$ we shall confine ourselves to the case where the summands in (3.1) satisfy the traditional u.a.n. condition (cf. Loève [5]).

Theorem 2. For any $\sigma \geqq 0$.

$$
\mathscr{L}\left(\Lambda_{n} \mid P_{n}^{(n)}\right) \rightarrow_{w} \mathscr{N}\left(-\frac{1}{2} \sigma^{2} ; \sigma^{2}\right)
$$

and

$$
\lim _{n \rightarrow \infty} \max _{1 \leqq i \leqq n} P_{n i}\left(\left|\log \left\{q_{n i}\left(X_{n i}\right) \mid p_{n i}\left(X_{n i}\right)\right\}\right| \geqq \varepsilon\right)=0
$$

for every $\varepsilon>0$ iff for every $\varepsilon>0$

$$
\lim _{n \rightarrow \infty} \sum_{i=i}^{n} H^{2}\left(P_{n i}, Q_{n i}\right)=\frac{1}{4} \sigma^{2},
$$




$$
\begin{aligned}
& \lim _{n \rightarrow \infty} \sum_{i=1}^{n} Q_{n i}\left(q_{n i}\left(X_{n i}\right) / p_{n i}\left(X_{n i}\right) \geqq 1+\varepsilon\right)=0, \\
& \lim _{n \rightarrow \infty} \sum_{i=1}^{n} P_{n_{i}}\left(p_{n i}\left(X_{n i}\right) / q_{n i}\left(X_{n i}\right) \geqq 1+\varepsilon\right)=0,
\end{aligned}
$$

or equivalently, iff (3.4) holds and for every $\varepsilon>0$

$$
\lim _{n \rightarrow \infty} \sum_{i=1}^{n} \int_{\left|q_{n i}-p_{n i}\right| \geqq \varepsilon p_{n i}}\left(q_{n i}^{1 / 2}-p_{n i}^{1 / 2}\right)^{2} \mathrm{~d} \mu_{n i}=0 .
$$

Proof. To simplify the notation we write $r_{n i}=q_{n i} / p_{n i}$. We first show that (3.5) and (3.6) are equivalent to (3.7). From

$$
\begin{gathered}
\sum_{i=1}^{n} \int_{\left|q_{n i}-p_{n i}\right| \geqq \varepsilon p_{n i}}\left(q_{n i}^{1 / 2}-p_{n i}^{1 / 2}\right)^{2} \mathrm{~d} \mu_{n i} \\
=\sum_{i=1}^{n}\left\{\int_{r_{n i} \geqq 1+\varepsilon} q_{n i}\left(1-r_{n i}^{-1 / 2}\right)^{2} \mathrm{~d} \mu_{n i}+\int_{r_{n i} \leqq 1-\varepsilon} p_{n i}\left(1-r_{n i}^{1 / 2}\right)^{2} \mathrm{~d} \mu_{n i}\right\}
\end{gathered}
$$

we obtain the double inequality

$$
\begin{gathered}
\left\{1-(1+\varepsilon)^{-1 / 2}\right\}^{2} \sum_{i=1}^{n} Q_{n i}\left(r_{n i}\left(X_{n i}\right) \geqq 1+\varepsilon\right) \\
+\left\{1-(1-\varepsilon)^{1 / 2}\right\}^{2} \sum_{i=1}^{n} P_{n i}\left(r_{n i}^{-1}\left(X_{n i}\right) \geqq(1-\varepsilon)^{-1}\right) \\
\leqq \sum_{i=1}^{n} \int_{\left|q_{n i}-p_{n i}\right| \geqq \varepsilon p_{n i}}\left(q_{n i}^{1 / 2}-p_{n i}^{1 / 2}\right)^{2} \mathrm{~d} \mu_{n i} \\
\leqq \sum_{i=1}^{n} Q_{n i}\left(r_{n i}\left(X_{n i}\right) \geqq 1+\varepsilon\right)+\sum_{i=1}^{n} P_{n i}\left(r_{n i}^{-1}\left(X_{n i}\right) \geqq(1-\varepsilon)^{-1}\right)
\end{gathered}
$$

and the equivalence of (3.5) and (3.6) to (3.7) is immediate.

Next we note that both (3.2), (3.3) and (3.4), (3.5), (3.6) imply $\left\{P_{n}^{(n)}\right\} \triangle \triangleright\left\{Q_{n}^{(n)}\right\}$ (cf. Corollary 1).

The remainder of the proof relies on the normal convergence theorem (cf. Loève [5]). According to an equivalent form of this theorem (3.2) and (3.3) are equivalent to

$$
\begin{gathered}
\lim _{n \rightarrow \infty} \sum_{i=1}^{n} P_{n i}\left(\left|\log r_{n i}\left(X_{n i}\right)\right| \geqq \delta\right)=0 \text { for every } \delta>0, \\
\lim _{\delta \downarrow 0} \lim _{n \rightarrow \infty} \sum_{i=1}^{n} \int_{\left|\log r_{n i}\right| \leqq \delta}\left(\log r_{n i}\right) \mathrm{d} P_{n i}=-\frac{1}{2} \sigma^{2},
\end{gathered}
$$

$$
\lim _{\delta \nmid 0} \lim _{n \rightarrow \infty} \sum_{i=1}^{n}\left\{\int_{\left|\log r_{n i}\right| \leqq \delta}\left(\log r_{n i}\right)^{2} \mathrm{~d} P_{n i}-\left(\int_{\left|\log r_{n i}\right| \leqq \delta}\left(\log r_{n i}\right) \mathrm{d} P_{n i}\right)^{2}\right\}=\sigma^{2} .
$$


By the contiguity of $\left\{P_{n}^{(n)}\right\}$ and $\left\{Q_{n}^{(n)}\right\}$ and (2.1) the condition (3.8) is equivalent to (3.5) and (3.6) and hence to (3.7). Henceforth we assume (3.7), (3.8) and $\left\{P_{n}^{(n)}\right\} \triangleleft \triangleright$ $\triangle \triangleright\left\{Q_{n}^{(n)}\right\}$. We still have to show that (3.4) is equivalent to (3.9) and (3.10).

Let $0<\delta<1$. For $\left|\log r_{n i}\right| \leqq \delta$ we have the expansion

$$
\begin{gathered}
\log r_{n i}=2 \log \left\{1+\left(q_{n i}^{1 / 2}-p_{n i}^{1 / 2}\right) p_{n i}^{-1 / 2}\right\} \\
=2\left(q_{n i}^{1 / 2}-p_{n i}^{1 / 2}\right) p_{n i}^{-1 / 2}-\left(q_{n i}^{1 / 2}-p_{n i}^{1 / 2}\right)^{2} p_{n i}^{-1}\left(1+\varrho_{n i \delta}\right)
\end{gathered}
$$

with $\left|\varrho_{n i \delta}\right|<2 \delta$. Thus

$$
\begin{gathered}
\int_{\left|\log r_{n i}\right| \leqq \delta}\left(\log r_{n i}\right) p_{n i} \mathrm{~d} \mu_{n i} \\
=-2 \int_{\left|\log r_{n i}\right| \leqq \delta}\left(q_{n i}^{1 / 2}-p_{n i}^{1 / 2}\right)^{2} \mathrm{~d} \mu_{n i}+\int_{\left|\log r_{n i}\right| \leqq \delta}\left(q_{n i}-p_{n i}\right) \mathrm{d} \mu_{n i} \\
-\int_{\mid \log r_{n i \mid} \leqq \delta} \varrho_{n i \delta}\left(q_{n i}^{1 / 2}-p_{n i}^{1 / 2}\right)^{2} \mathrm{~d} \mu_{n i} .
\end{gathered}
$$

Since by (3.7)

$$
\lim _{n \rightarrow \infty}\left\{\sum_{i=1}^{n} \int_{\left|\log r_{n i}\right| \leqq \delta}\left(q_{n i}^{1 / 2}-p_{n i}^{1 / 2}\right)^{2} \mathrm{~d} \mu_{n i}-\sum_{i=1}^{n} H^{2}\left(P_{n i}, Q_{n i}\right)\right\}=0
$$

and by (3.8), $\left\{P_{n}^{(n)}\right\} \triangleleft \triangleright\left\{Q_{n}^{(n)}\right\}$ and (2.1)

$$
\sum_{i=1}^{n} \int_{\left|\log r_{n i}\right| \leqq \delta}\left(q_{n i}-p_{n i}\right) \mathrm{d} \mu_{n i}=-\sum_{i=1}^{n} \int_{\left|\log r_{n i}\right|>\delta}\left(q_{n i}-p_{n i}\right) \mathrm{d} \mu_{n i} \rightarrow 0
$$

for $n \rightarrow \infty$, we have

$$
\begin{aligned}
\underset{\delta \downarrow 0}{\lim } \limsup _{n \rightarrow \infty} & \left|\sum_{i=1}^{n} \int_{\left|\log r_{n i}\right| \leqq \delta}\left(\log r_{n i}\right) \mathrm{d} P_{n i}+2 \sum_{i=1}^{n} H^{2}\left(P_{n i}, Q_{n i}\right)\right| \\
& \leqq \lim _{\delta \downarrow 0} \limsup _{n \rightarrow \infty} 2 \delta \sum_{i=1}^{n} H^{2}\left(P_{n i}, Q_{n i}\right)=0,
\end{aligned}
$$

where we have used (1.6). Similarly,

$$
\begin{aligned}
& \lim _{\delta \downarrow 0} \limsup _{n \rightarrow \infty} \sum_{i=1}^{n}\left\{\int_{\left|\log r_{n i}\right| \leqq \delta}\left(\log r_{n i}\right) \mathrm{d} P_{n i}\right\}^{2} \\
& \leqq \lim _{\delta \downarrow 0} \limsup _{n \rightarrow \infty} \delta \sum_{i=1}^{n}\left|\int_{\left|\log r_{n i}\right| \leqq \delta}\left(\log r_{n i}\right) \mathrm{d} P_{n i}\right| \\
& \leqq \lim _{\delta \downarrow 0} \limsup _{n \rightarrow \infty} \delta(2+2 \delta) \sum_{i=1}^{n} H^{2}\left(P_{n i}, Q_{n i}\right)=0 .
\end{aligned}
$$


Finally (3.11) implies that for $\left|\log r_{n i}\right| \leqq \delta<1$

$$
\left(\log r_{n i}\right)^{2}=4\left(q_{n i}^{1 / 2}-p_{n i}^{1 / 2}\right)^{2} p_{n i}^{-1}+\bar{\varrho}_{n i \delta}\left(q_{n i}^{1 / 2}-p_{n i}^{1 / 2}\right)^{2} p_{n i}^{-1}
$$

with $\left|\bar{\varrho}_{n i \delta}\right|<10 \delta$. Hence, in view of (3.7) and (1.6),

$$
\lim _{\delta \downarrow 0} \limsup _{n \rightarrow \infty}\left|\sum_{i=1}^{n} \int_{\left|\log r_{n i}\right| \leqq \delta}\left(\log r_{n i}\right)^{2} \mathrm{~d} P_{n i}-4 \sum_{i=1}^{n} H^{2}\left(P_{n i}, Q_{n i}\right)\right|=0 \text {. }
$$

The equivalence of (3.4) to (3.9) and (3.10) is now an immediate consequence of (3.12), (3.13) and (3.14). The theorem is proved.

In the one sample case where, for each $n, X_{n 1}, \ldots, X_{n n}$ are identically distributed, condition (3.3) is implied by (3.2) and Theorem 2 slightly simplifies. This remains true in the $k$ sample case $(k \geqq 2)$ provided all sample sizes tend to infinity.

The first part of the proof of Theorem 2 also shows that the conditions (2.3) and (2.5) in Corollary 1 may be replaced by the single condition

$$
\lim _{n \rightarrow \infty} \sum_{i=1}^{n} \int_{\left|q_{n i}-p_{n i}\right| \geqq c_{n} p_{n i}}\left(q_{n i}^{1 / 2}-p_{n i}^{1 / 2}\right)^{2} \mathrm{~d} \mu_{n i}=0 \text { whenever } \quad c_{n} \rightarrow \infty .
$$

The proof of Theorem 2 could also be given in a more roundabout way. Introducing the r.v.'s

$$
W_{n i}=2\left\{q_{n i}\left(X_{n i}\right) / p_{n i}\left(X_{n i}\right)\right\}^{1 / 2}-2, \quad i=1, \ldots, n ; n=1,2, \ldots,
$$

one shows that $\mathscr{L}\left(\sum_{i=1}^{n} W_{n i} \mid P_{n}^{(n)}\right) \rightarrow_{w} \mathcal{N}\left(-\frac{1}{4} \sigma^{2} ; \sigma^{2}\right)$ iff $\mathscr{L}\left(\Lambda_{n} \mid P_{n}^{(n)}\right) \rightarrow_{w} \mathcal{N}\left(-\frac{1}{2} \sigma^{2} ; \sigma^{2}\right)$, provided the respective u.a.n. conditions are satisfied. It is then not difficult to prove that the weak convergence of $\sum_{i=1}^{n} W_{n i}$ and the u.a.n. condition on the summands are equivalent to (3.4) and (3.7). In this proof (3.7) appears as the Lindeberg condition in the central limit theorem applied to $\sum_{i=1}^{n} W_{n i}$.

The equivalence of both weak convergence results has first been proved by Le Cam ([3], [4]). The initial assumptions $\lim _{n \rightarrow \infty} \sup _{1 \leq i \leq n} H^{2}\left(P_{n i}, Q_{n i}\right)=0$ and $\underset{n \rightarrow \infty}{\limsup }\left\|P_{n}^{(n)}-Q_{n}^{(n)}\right\|<1$ made by Le Cam are not restrictive since they are implied by our condition (3.7) and the contiguity of $\left\{P_{n}^{(n)}\right\}$ and $\left\{Q_{n}^{(n)}\right\}$, respectively. One part of this proof is also contained in Hájek \& Šidák [1]. 


\section{References}

[1] HÁJEK, J. - ŠIdÁK, Z. (1967). "Theory of rank tests”. Acađemic Press, New York.

[2] Le CAM, L. (1960). Locally asymptotically normal families of distributions. Univ. California Publ. Statist., 3, 37-98, University of California Press.

[3] LE CAM, L. (1966). Likelihood functions for large numbers of independent observations. Research papers in statistics (Festschrift for J. Neyman), 167-187, F. N. David (ed.), Wiley, New York.

[4] Le CaM, L. (1969). Théorie asymptotique de la décision statistique. Les Presses de l'Université de Montréal.

[5] Lok̀ve, M. (1963). "Probability theory (3rd ed.)". Van Nostrand, New York.

[6] Roussas, G. G. (1972). Contiguity of probability measures: some applications in statistics, Cambridge University Press.

[7] Witting, H. - Nölle G. (1970). "Angewandte mathematische Statistik”. Teubner, Stuttgart.

(1) CATH. UNIV. NYMEGEN, NYMEGEN, THE NETHERLANDS

(2) CENTRAAL REKENINSTITUUT DER RIJKSUNIVERSITEIT, LEIDEN, THE NETHERLANDS

Received October 1975 